\title{
Incorporating photomask shape uncertainty in computational lithography
}

\author{
Xiaofei $\mathrm{Wu}^{\mathrm{a}, \mathrm{c}}$, Shiyuan Liu ${ }^{\mathrm{b}}$, Andreas Erdmann ${ }^{\mathrm{c}}$, and Edmund Y. Lama \\ amaging Systems Laboratory, Department of Electrical and Electronic Engineering, \\ The University of Hong Kong, Pokfulam, Hong Kong \\ bState Key Laboratory of Digital Manufacturing Equipment and Technology, \\ Huazhong University of Science and Technology, Wuhan, China \\ ${ }^{c}$ Fraunhofer Institute for Integrated Systems and Device Technology, \\ Schottkystrasse 10, 91058 Erlangen, Germany
}

\begin{abstract}
The lithographic performance of a photomask is sensitive to shape uncertainty caused by manufacturing and measurement errors. This work proposes incorporating the photomask shape uncertainty in computational lithography such as inverse lithography. The shape uncertainty of the photomask is quantitatively modeled as a random field in a level-set method framework. With this, the shape uncertainty can be characterized by several parameters, making it computationally tractable to be incorporated in inverse lithography technique (ILT). Simulations are conducted to show the effectiveness of using this method to represent various kinds of shape variations. It is also demonstrated that incorporating the shape variation in ILT can reduce the mask error enhancement factor (MEEF) values of the optimized patterns, and improve the robustness of imaging performance against mask shape fluctuation.
\end{abstract}

Keywords: Computational lithography, inverse lithography, shape uncertainty, random field

\section{INTRODUCTION}

The continuous delay of alternative lithographic techniques such as extreme ultraviolet lithography drives the extension of ArF immersion lithography to much more advanced technological nodes. To cope with the challenges of extremely high resolution in these nodes, holistic optimization becomes necessary for the lithographic system, and computational lithography such as source mask optimization and inverse lithography technique (ILT) are strong candidates. ${ }^{1,2}$ While computational lithography relies on lithographic simulation to faithfully model the lithographic system, the patterning process from the photomask to wafer is a stochastic process, and significant noise and parameter uncertainties make an accurate prediction difficult. ${ }^{3,4}$

One category of such uncertainties in the lithography system can be the photomask shape uncertainty. ${ }^{5}$ The mask in lithography is made by e-beam writing, and can be imperfect with significant errors on the critical dimension (CD), which can influence the intra-field CD uniformity on the final wafer. ${ }^{6}$ The CD non-uniformity, which is the error between printed mask CD and target CD during the mask making process, can be caused by the e-beam exposure, the e-beam resist process and the etch process. Previous studies also show that the lens heating effect can cause mask shape deviation and best focus shift in the patterning process. ${ }^{7}$ The error can include long-range effects, such as CD uniformity and pattern loading, and short-range effects, such as proximity and linearity. Efforts have been made to reduce such errors, such as the mask process correction (MPC) and the dry etching loading effect correction. High-frequency errors such as line-edge roughness and line-width roughness also gain much interest, and efforts have been made to explore their influence on the lithographic performance and control them to an acceptable level. ${ }^{8}$ However, the low-frequency errors are the dominating effects that are the main challenge for further feature size shrinkage. ${ }^{9}$

Further author information: (Send correspondence to Edmund Y. Lam)

Edmund Y. Lam: E-mail: elam@eee.hku.hk, Telephone: +852 2241-5942

Optical Microlithography XXIX, edited by Andreas Erdmann and Jongwook Kye, Proc. of SPIE Vol. 9780, 97800Q · (C) 2016 SPIE · CCC code: 0277-786X/16/\$18 - doi: 10.1117/12.2220278 
In this work, we explore a method to incorporate random mask shape uncertainty during the mask optimization in inverse lithography. The aim is to derive a mask pattern that is robust against the shape uncertainty that is unavoidable, indicating that the printed wafer pattern could be less sensitive to shape errors on the mask. This concept has been proposed to integrate the photomask CD errors into an optical proximity correction (OPC) modeling and correction. ${ }^{6}$ However, the extension to inverse lithography introduces new issue considering the large amount of pixel variables to optimize. The concept can also be considered as an extension of the mask error enhancement factor (MEEF) reduction, which is an objective in source mask optimization (SMO). ${ }^{10,11}$ However, the constraint of MEEF in SMO usually consider uniform mask shape errors, and the shape variation here can be randomly distributed.

This work uses a random field method to represent the mask pattern in optical lithography, and inverse lithography is performed in a level-set framework. ${ }^{12}$ In this approach, the mask shape is defined as the zeros of its corresponding signed distance function. The random field method is a statistical technique to represent highly correlated multivariate random vectors. By using a Karhunen-Loève (K-L) expansion of the covariance function of the random field, the random vector is expressed with the eigenfunctions and the eigenvalues. Since the eigenvalues decrease rapidly, only several eigenfunctions are preserved for the representation. Thus, the multi-dimensional vectors are expressed with only several variables. When the signed distance function is added with the random field, it can be used to represent a mask pattern with random shape variations. With this representation, optimization algorithm can be used to derive a mask pattern that is robust against such variations in inverse lithography.

In the subsequent sections, we first present the random field method and use it to represent the mask shape variations. Then, we introduce a robust optimization algorithm and its acceleration to incorporate the shape uncertainty into inverse lithography in section 3. After that, we provide simulation results to show the shape variations the method can represent and the effectiveness of the optimization algorithm in section 4, which are followed by some discussions and conclusion in section 5 .

\section{RANDOM FIELD METHOD TO MODEL SHAPE UNCERTAINTY}

To incorporate the photomask shape variations into a mask optimization process, we first need a method to represent the shape variations. In the mask optimization work here, this is done by using a random field to describe a stochastic level-set function. In the level-set based inverse lithography, ${ }^{13}$ the mask is expressed by a level-set function $\phi(\mathbf{x}, t)$

$$
M(\mathbf{x})= \begin{cases}1, & \text { for } \phi(\mathbf{x}, t) \leq 0 \\ 0, & \text { for } \phi(\mathbf{x}, t)>0\end{cases}
$$

where $\mathbf{x}$ is the spatial coordinate, the shape of the mask is implicitly represented as the zeros of the level-set function. A time dimension $t$ is introduced to indicate the mask boundary evolution, and the level-set function changes according to the time during the optimization process.

To represent mask patterns with shape fluctuation, a random field method with K-L expansion is used to describe the level-set function. Let $g(\mathbf{x}, \theta)$ be a random field defined on a spatial domain $\mathbf{x}$, where $\theta$ denotes an element of the random sample space. The random field can be expressed by the K-L expansion ${ }^{14}$

$$
g(\mathbf{x}, \theta)=\bar{g}(\mathbf{x})+\sum_{i=1}^{+\infty} \sqrt{\lambda_{i}} \psi_{i}(\mathbf{x}) \xi_{i}(\theta)
$$

where $\bar{g}(\mathbf{x})$ is the mean, $\xi_{i}(\theta)$ are the random variables to represent the uncertainty, $\lambda_{i}$ and $\psi_{i}(\mathbf{x})$ are the $i$ th eigenvalue and eigenfunction obtained from the following integral equation

$$
\int C\left(\mathbf{x}_{1}, \mathbf{x}_{2}\right) \psi_{i}\left(\mathbf{x}_{1}\right) d \mathbf{x}_{1}=\lambda_{i} \psi_{i}\left(\mathbf{x}_{2}\right),
$$

where $C\left(\mathbf{x}_{1}, \mathbf{x}_{2}\right)$ is the spatial covariance function to define the random field. The random variables $\xi_{i}(\theta)$ are orthogonal to each other with zero mean and unit variance, that is,

$$
E\left[\xi_{i}(\theta)\right]=0, \text { and } E\left[\xi_{i}(\theta) \xi_{j}(\theta)\right]=\delta_{i j} .
$$




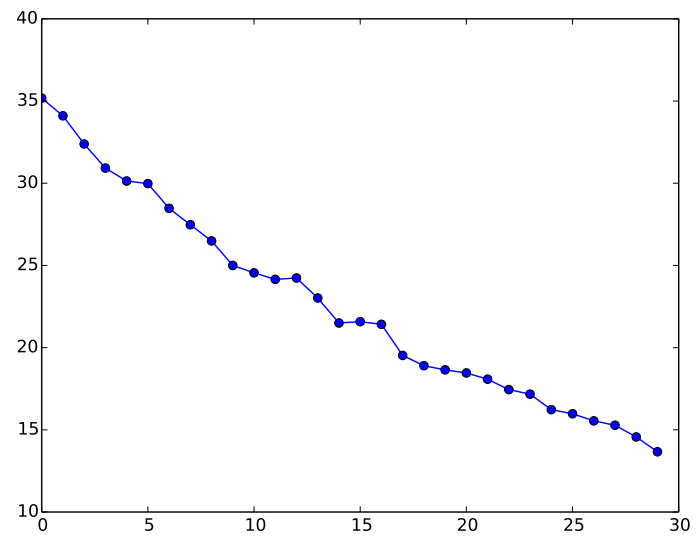

(a) The eigenvalues of the random field, and that their values are decreasing
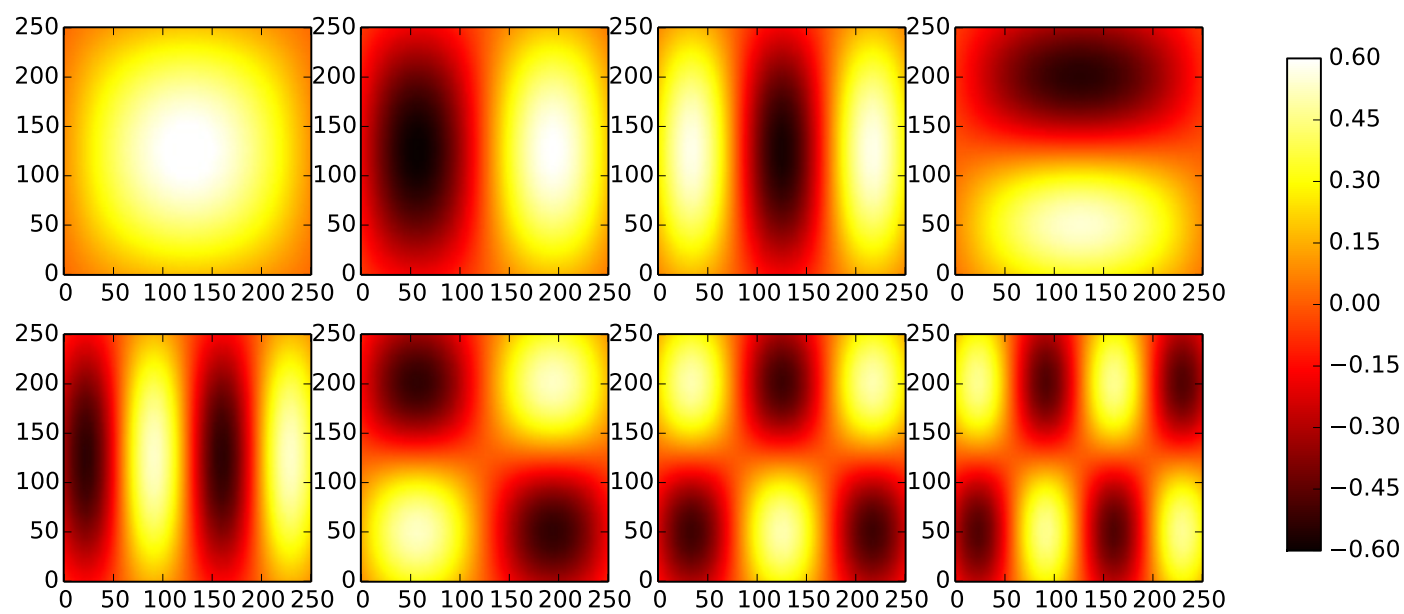

(b) The first 8 eigenfunctions of the random field, where they have different distributions so that they can represent random shape variations.

Figure 1. Illustration of the random field to represent the shape variations

With this representation, the quantification of the multivariate random field is transferred to the random variables $\xi(\theta)$, whose dimension is the same as the number of eigenfunctions of the covariance matrix. By truncating the eigenfunctions in Eq. (2), a reduced order K-L expansion can be achieved to represent the random field, indicating a much smaller dimension of random variables. Considering the rapidly decreasing values of the eigenvalues, this can be possible and will not significantly reduce the accuracy of the random field.

Thus, we can construct a random field to model the random shape variations of the mask patterns. The level-set function corresponding to the mask is set as the mean of the random field, and then a random level-set function can be expressed as

$$
\phi(\mathbf{x}, \theta)=\bar{\phi}(\mathbf{x})+\sum_{i=1}^{K} \sqrt{\lambda_{i}} \psi_{i}(\mathbf{x}) \xi_{i}(\theta)
$$

where $K$ is the truncation order of the K-L expansion. A predefined covariance matrix can be defined to quantify the uncertainty, and this covariance matrix is defined as an exponential function of the distance between two points

$$
C=\exp \left(-\frac{\left\|\mathbf{x}_{1}-\mathbf{x}_{2}\right\|}{d}\right)
$$


where $d$ is variable to control the correlation of two points, and $\|\cdot\|$ is the Euclidean distance. The mask patterns corresponding to the level-set functions then have random shape variation from the original mask patterns, and this uncertainty can be represented by $K$ random variables.

Figure 1 shows the eigenvalues and first eight eigenfunctions of a random field described above. As shown in Fig. 1(a), the eigenvalues of the random field decrease quickly, and thus only a part of the eigenvalues are needed to represent the random field. We also observe that the eigenfunctions in Fig. 1(b) have different distributions from each other, and thus their superposition can be used to simulate random shape changes of the mask patterns.

\section{INVERSE LITHOGRAPHY WITH SHAPE VARIATIONS}

In this section, we introduce an optimization algorithm to obtain a pattern that is robust to mask shape variations by using the random field representation. As shown in Eq. (5), a random field $\phi(\mathbf{x}, \theta)$ can be used to represent a random mask with random shape variations. By assuming a normal distribution of each of the random variable in the representation

$$
p\left(\xi_{i}(\theta)\right)=\frac{1}{\sigma \sqrt{2 \pi}} \exp \left(-\frac{\xi_{i}^{2}(\theta)}{2 \sigma^{2}}\right)
$$

where $\sigma$ is the variation, we can generate a series of mask patterns with shape variations. Then, we define the mean pattern fidelity of these mask patterns as the objective function as

$$
\begin{aligned}
\mu_{\mathcal{L}}\{\phi(\mathbf{x}, \theta)\} & =\mathbf{E}\left\{\mathcal{L}\left\{\tilde{\mathbf{T}}[\phi(\mathbf{x}, \theta)], I_{t}(\mathbf{x})\right\}\right\}, \\
& =\sum_{\theta}\left\{\prod_{i=1}^{N}\left\{p\left(\xi_{i}(\theta)\right)\right\} \mathcal{L}\left\{\tilde{\mathbf{T}}[\phi(\mathbf{x}, \theta)], I_{t}(\mathbf{x})\right\}\right\},
\end{aligned}
$$

where $\tilde{\mathbf{T}}[\cdot]$ denotes the pattern transfer from the level-set function to the output pattern on the wafer, $\mathcal{L}$ is the distance of output pattern to the target one, which are introduced in our previous work. ${ }^{15,16}$ The objective function $\mu_{\mathcal{L}}$ is the expectation of the pattern fidelities over the random space denoted by $\theta$. As the truncation of the K-L expansion is used to represent the random field, the statistical average is computed over several random variables, instead of all the pixel variables in inverse lithography. This can significantly reduce the computation cost, and make it possible to incorporate the shape uncertainty into the optimization.

Even with this approximation, the optimization can still be computationally expensive since it needs to be conducted over many realizations of the random variables, especially using the gradient-based algorithms. For instance, if we set $S$ realizations for each random variable in the random space $\theta$, and the truncation order is $N$, a total number of $N^{S}$ realizations need to be computed in the optimization. To tackle this issue, we introduce a minimax algorithm to further accelerate the algorithm. We first calculate the pattern fidelities for all the realizations corresponding to the random variables, and then we sort them in a decreasing order and find the largest several ones. Instead of minimizing the expectation of pattern fidelities corresponding to all the realizations, we set the objective function to minimize the average of several largest ones as

$$
\mu_{\mathcal{L}}^{\prime}\{\phi(\mathbf{x}, \theta)\}=\sum_{j=1}^{P} p_{j}\left(\phi_{j}(\mathbf{x}, \theta)\right) \mathcal{L}\left\{\tilde{\mathbf{T}}\left[\phi_{j}(\mathbf{x}, \theta)\right], I_{t}(\mathbf{x})\right\},
$$

where $P$ is the number of realizations to minimize, $\phi_{j}(\mathbf{x}, \theta)$ is the $j$ th realization, and $p_{j}\left(\phi_{j}(\mathbf{x}, \theta)\right)$ is the probability corresponding to the realization $\phi_{j}$. In this way, though we still need to compute the pattern fidelities for all the mask patterns, the computation of gradient reduces from $N^{S}$ to $P$, which again can improve the computational efficiency. Thus, an optimized mask can be obtained by solving an inverse problem using gradient-based algorithm ${ }^{15,16}$

$$
M_{t}(\mathbf{x})=\underset{0 \leq M(\mathbf{x}) \leq 1}{\arg \min } \mu_{\mathcal{L}}^{\prime}\{\phi(\mathbf{x}, \theta)\} .
$$



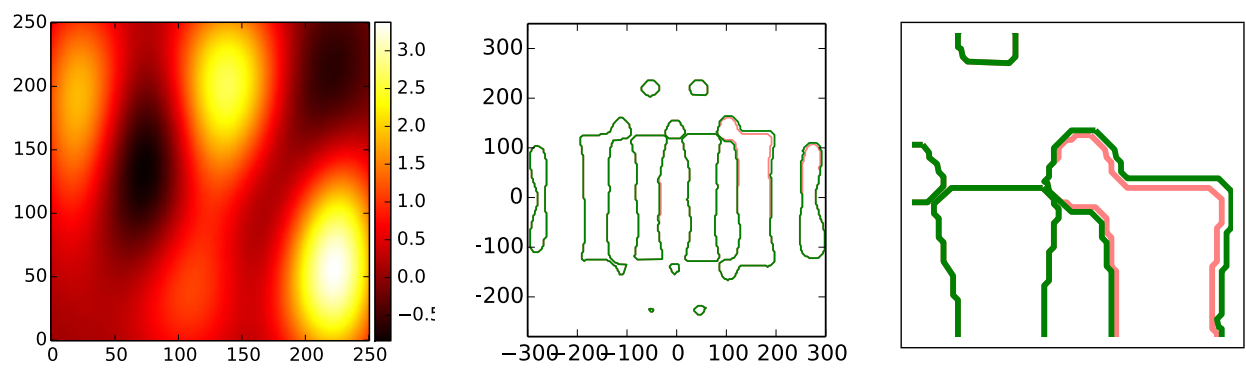

(a) random field with a size of $250 \times 250$, and the magnitudes are in $[-2,2]$
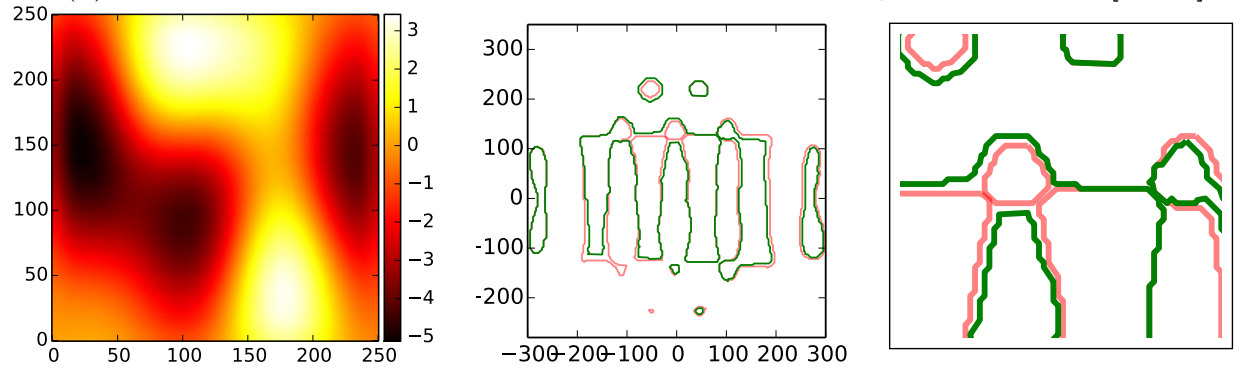

(b) random field with a size of $250 \times 250$, and the magnitudes are in $[-4,4]$
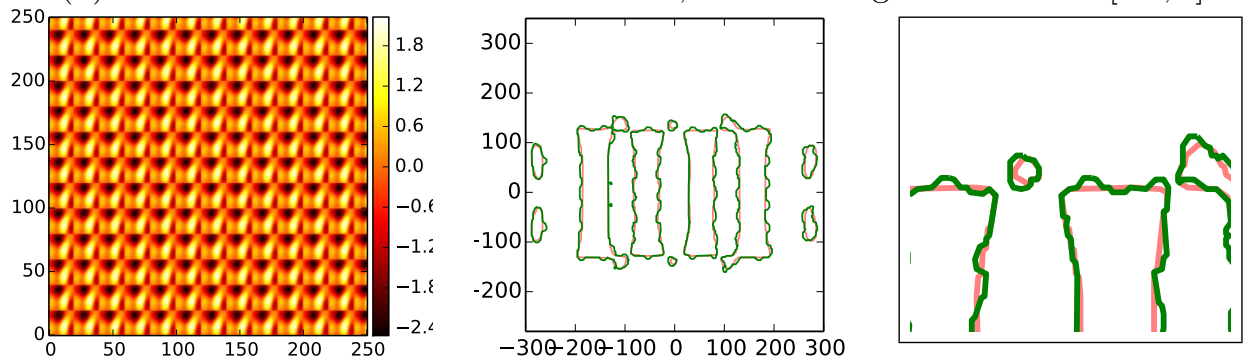

(c) random field with a size of $20 \times 20$, and the magnitudes are in $[-2,2]$

Figure 2. Illustration of the several realizations of random mask patterns. In each row, from the left to the right show a realization of a random field, the contour of the original mask (in red) and the one for the mask pattern with shape variations (in green), and a magnified view of the differences.

\section{SIMULATIONS AND RESULTS}

In the simulations, we demonstrate the shape variations the random field can represent, and analyze their impact on the lithographic performance. We also perform mask optimization on two typical test patterns and make comparisons of inverse lithography with and without considering the shape uncertainties by performing mask optimization on the test patterns.

In Fig. 2, several realizations of random field and their corresponding mask patterns with variations are illustrated. The level-set function of an arbitrary mask pattern is set as the mean of the random field. In this example, it is represented as a $250 \times 250$ matrix, and the contour is shown as red curves in the middle panel of each row. Each row of the figure shows the distribution of the random field realization, the corresponding mask patterns, and a magnified illustration of the variations from the left to the right. The mask pattern with shape variation is shown as green contours to make a comparison of the mean mask.

In different rows of the figure, we change the parameters of the random field, such as the magnitude of the random variables and the sizes of the random field so that different mask shape variations can be simulated. In the first two rows, the random field is set as the same size of the level-set function, while the magnitudes of the random variables are $[-2,2]$ for the first row, and $[-4,4]$ for the second row. Obviously, the shape variation in the second row is larger that that in the first row. In the third row, we change the size of random field to $20 \times 20$ and tile them together to the scale of the level-set function to simulate the shape variations. Here, we 

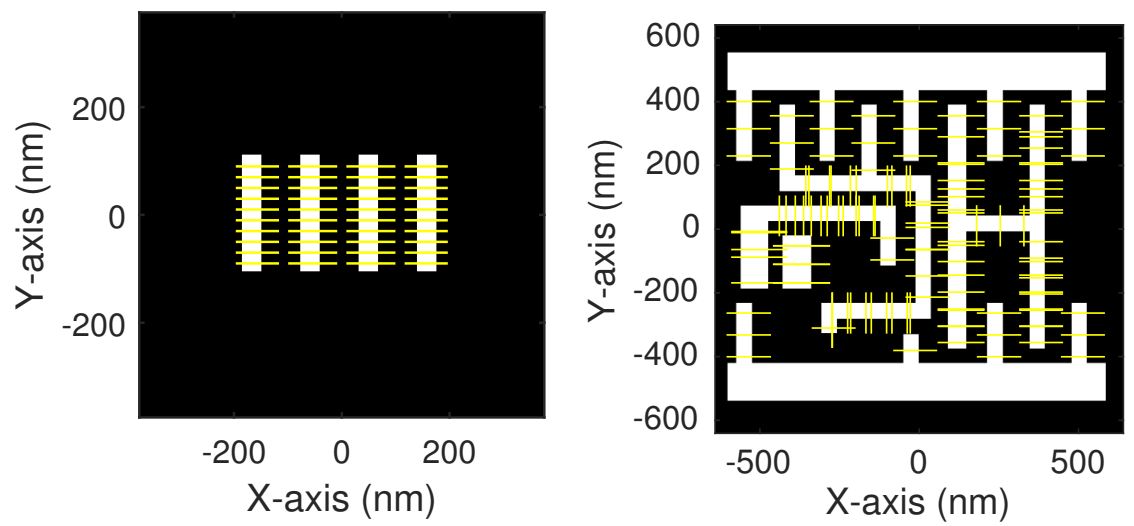

Figure 3. The test target patterns in the mask optimization. The left one is a four bar pattern, and the right is a more complex one.

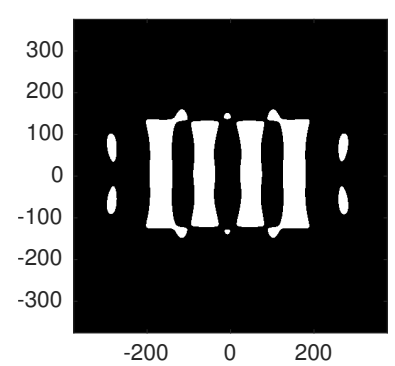

(a) optimized pattern with traditional ILT

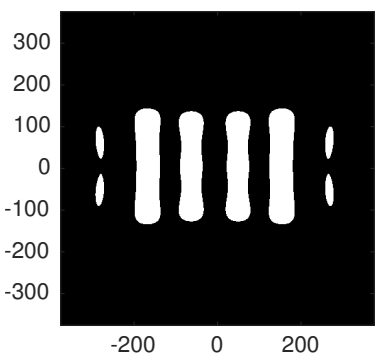

(c) optimized pattern with proposed ILT

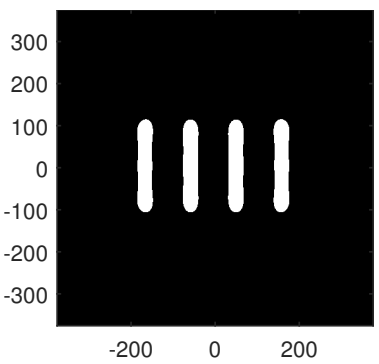

(b) resist pattern for pattern (a)

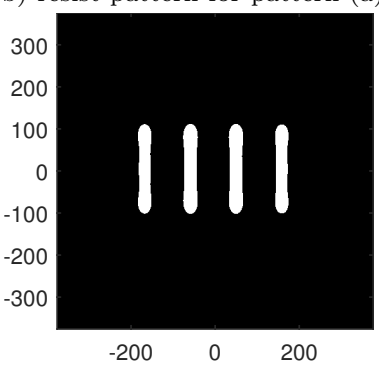

(d) resist pattern for pattern (c)

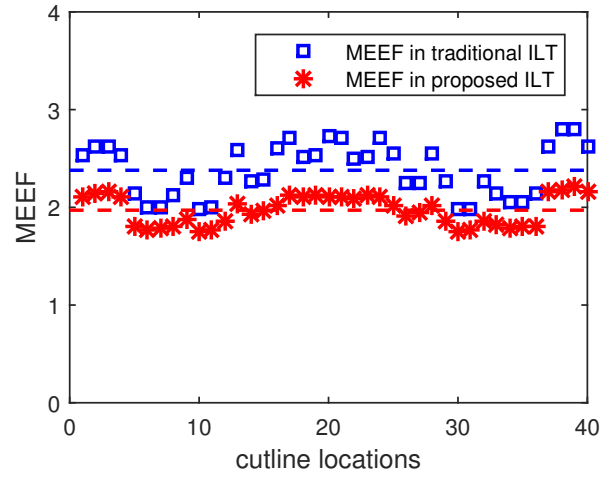

(e) comparison of MEEF values

Figure 4. Comparison of the optimized mask patterns with and without considering the shape variations. The upper row shows the optimized mask patterns, the corresponding resist pattern at best focus plane with the traditional ILT, and those for the proposed ILT are in the lower row. The right panel depicts the measure MEEF values for the two optimized pattern.

use repeated same realizations to tile them together, but note that the shape variation at different places of the mask can still be different since it is feature dependent. Making a comparison of the shape changes with that shown in the first row, the deviations to the mean mask have a higher frequency. Thus, the random field can be adjusted to simulate different kinds of shape variations on the mask.

Then we conduct mask optimization with two test patterns, including a four bar pattern on the left, and a more complex one on the right. They are both represented by a $500 \times 500$ matrix, and the pixel sizes are $1.5 \mathrm{~nm}$ and $2.4 \mathrm{~nm}$, respectively. The yellow lines in the figure depict the cutline locations to measure the mask error enhancement errors (MEEF) and CD errors. The optimization is done for ArF immersion lithographic system with $\mathrm{NA}=1.35$, and the wavelength is $193 \mathrm{~nm}$. The illumination source is set as quasar with an opening angle of $45^{\circ}$, and the inner and outer radii are 0.62 and 0.92 . The inverse lithography is performed with two methods, including a traditional methods without considering the shape variations, and the proposed method with the consideration introduced in the section above. In order to simplify the calculation, the mask optimization is performed in the best focus plane. The truncation order to represent the random field is set as $N=3$, and $S=5$ 


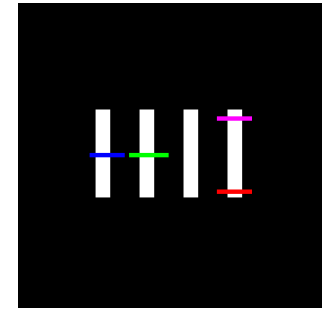

(a) cutlines to measure OPW

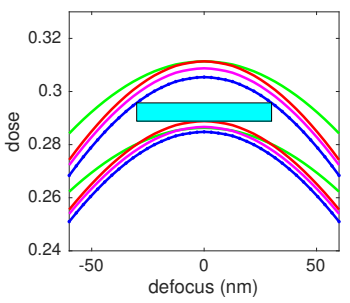

(b) OPW for pattern

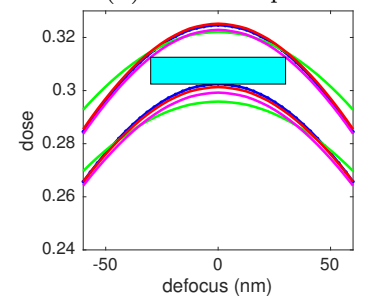

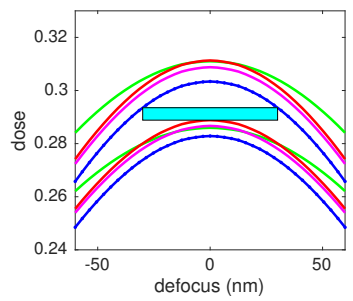
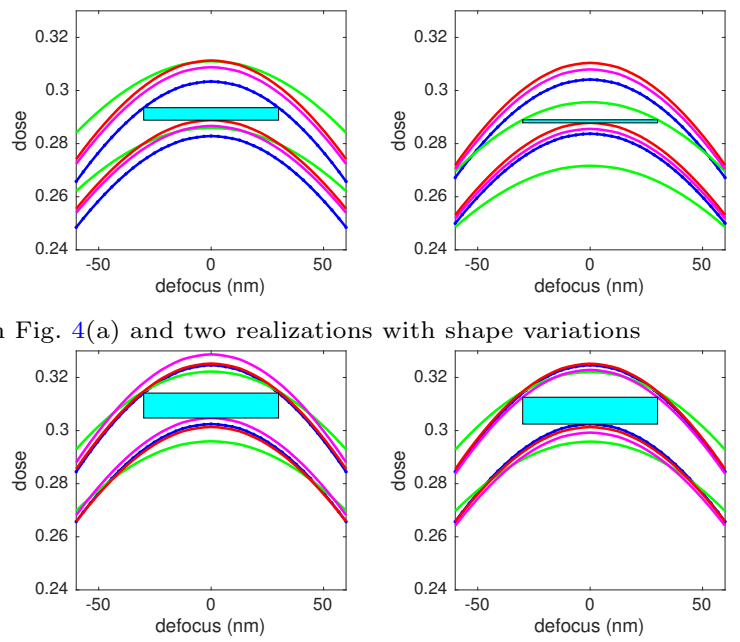

(c) OPW for pattern in Fig. 4(c) and two realizations with shape variations

Figure 5. Comparison of overlapping process window (OPW) sizes measured at four cutline locations shown in (a) for the four bar mask pattern. The three OPWs on the upper row corresponds to the result of the optimized pattern shown in Fig. 4(a) and two mask patterns with shape variations. The lower row shows similar OPWs for the optimized pattern for the proposed ILT.

for each random variable in the optimization. The number of realizations to compute the gradient $P$ is set as 5 . The convergence condition in both the optimization is set the same so that comparison can be done to evaluate the performance of the latter algorithm.

The optimization result for the four bar mask pattern is shown in Fig. 4. Two optimized mask patterns obtained with and without considering the shape variations are shown in Fig. 4(a) and 4(c). The corresponding output patterns at the best focus plane are shown in Fig. 4(b) and 4(d), and the measured MEEF values at the cutline locations are shown in $4(\mathrm{e})$. It is observed that the output patterns have similar imaging performance, but the optimized patterns are different regarding assistant features sizes and locations. Simultaneously, it is observed that the MEEF values obtained in the proposed ILT are much smaller than those obtained in traditional ILT. This means that the mask patterns obtained with the proposed ILT can be more robust to shape variations.

We also analyze how the shape uncertainty can affect the imaging performance in terms of overlapping process window (OPW) in Fig. 5. The cultine locations to measure the OPW of the first test pattern are shown in Fig. 5(a). The three panels in the upper row show the OPWs for the optimization pattern in Fig. 4(a), and two realizations of mask patterns with shape variations. The lower row shows similar OPWs for mask patterns obtained with the proposed ILT. The magnitude of the random variables to generate the shape differences is set as the same to make the comparison. It is demonstrated that the sizes of OPW for the mask patterns with shape variations can be much smaller in the traditional ILT. In the lower row, the sizes of OPW have a minor changes, indicating incorporation the shape variations in the optimization can reduce the sensitivity of OPW sizes to the mask errors.

Similarly, we depict the simulation results for the second mask pattern in Fig. 6. Again, we observe that the two mask patterns obtained with the two ILT methods have a significant difference. The locations of the assistant features shown in Fig 6(a) are different from those shown in Fig. 6(c). The MEEF values at the cutline locations shown in Fig. 3 for both the mask patterns are depicted in Fig. 6(e). Since this test pattern is more complicated, the MEEF values for both the optimized patterns are higher than those obtained for the first test case. We also observe that the MEEF values for the mask pattern with the proposed ILT are lower than those for the pattern obtained in traditional ILT considering both the largest value and the average one. This further demonstrates that the ILT method considering the shape variations can be helpful to reduce the MEEF values.

To further analyze the effectiveness of the proposed ILT method, we evaluate the CD uniformity of some mask patterns with shape fluctuation. For each of the optimized patterns in both test cases, 50 realizations of the random field are generated with fixed magnitude of the random variables. We show the computed mean 


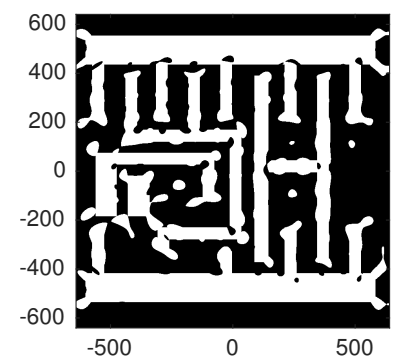

(a) traditionally optimized pattern

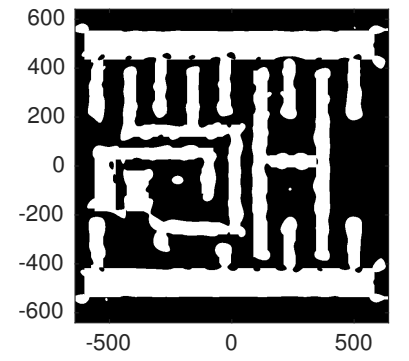

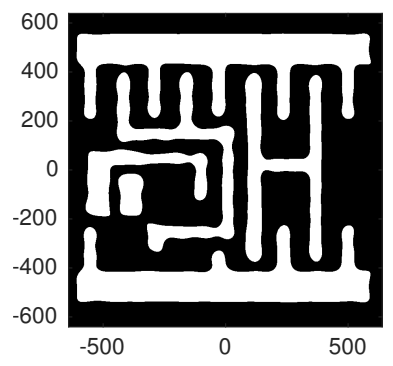

(b) resist pattern for pattern (a)

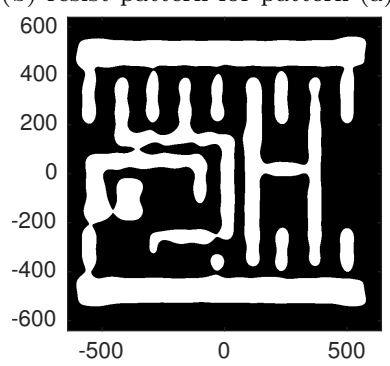

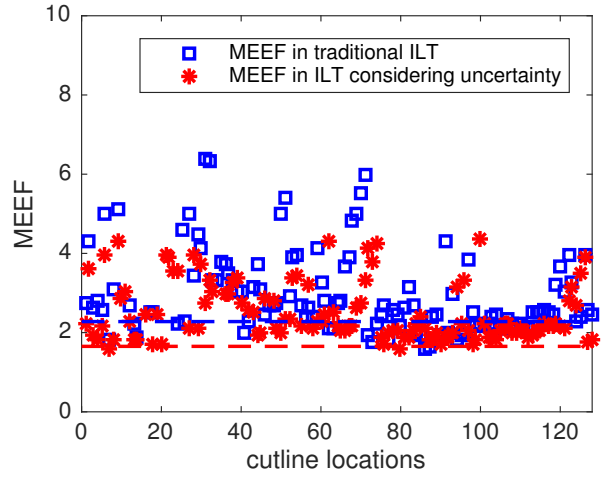

(e) comparison of MEEF values

(c) robust optimized pattern

(d) resist pattern for pattern (d)

Figure 6. The optimization result for a more complex mask pattern, where the result with the traditional ILT is shown in the upper row, and the one using the proposed method is shown in the lower row. Similar results of MEEF values is depicted on the right panel for both patterns.
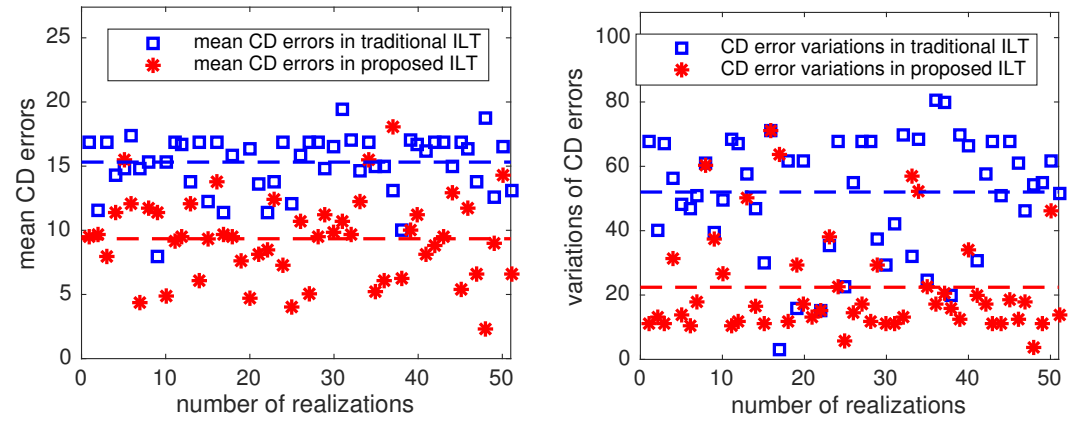

(a) CD uniformity analysis for test pattern 1
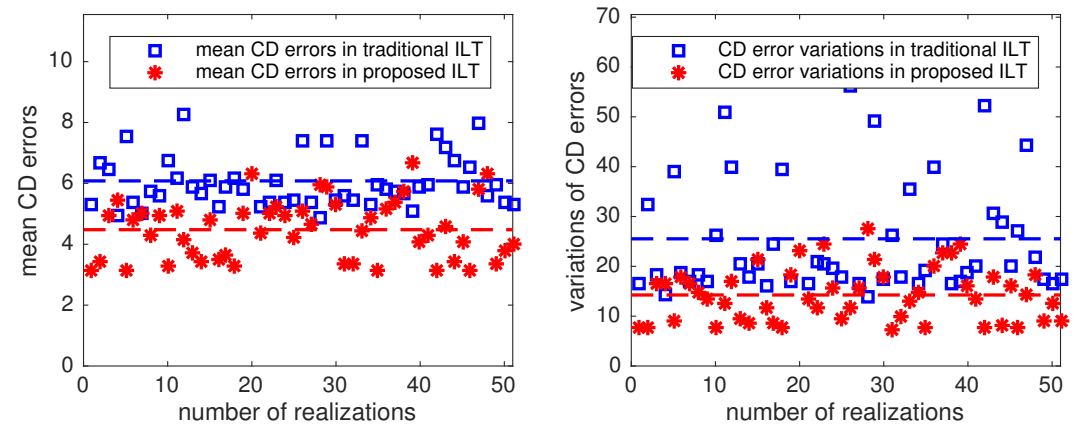

(b) CD uniformity analysis for test pattern 2

Figure 7. Evaluation of CD uniformity for 50 mask patterns with shape variations.

$\mathrm{CD}$ errors and $\mathrm{CD}$ variations in Fig. 7, where the upper row is for the first test pattern, and the lower row is for the second one. In the figure, we can see that the CD uniformity changes significantly with the randomly generated shape variations, even though the magnitude of the random variables are the same. In the upper row, the mean $\mathrm{CD}$ errors and $\mathrm{CD}$ variations for realizations corresponding to the optimized patterns generated with robust ILT are much smaller than the one obtained with the traditional ILT. The mean CD error reduces 
about $33 \%$, and the average CD error variation decreases for about $60 \%$ for the 50 realizations, which is quite a significant improvement of CD uniformity. Similar improvement of CD uniformity is also observed for the second test pattern regarding both the mean CD error and CD error variation. These results demonstrate that incorporating the shape variations in inverse lithography can improve the CD uniformity.

\section{CONCLUSIONS}

This work proposes to incorporate the mask shape variations into inverse lithography with a random field method. By taking the advantage of the K-L expansion, the random field method can represent photomask shape changes with only several parameters, and thus, it is computationally tractable to incorporate it into inverse lithography. Simulations are performed to show that the random field method can be used to model different shape fluctuation with different sizes and magnitudes. We also conduct simulations to perform inverse lithography with and without considering the shape variations and evaluate the optimization results in terms of MEEF values, OPW sizes, and CD uniformity. The simulation results demonstrate that inverse lithography with shape variations can reduce the sensitivity of the optimized pattern against shape fluctuations and can improve the CD uniformity simultaneously.

\section{ACKNOWLEDGMENTS}

This work was supported in part by the UGC Areas of Excellence project Theory, Modeling, and Simulation of Emerging Electronics, and by the State Key Lab of Digital Manufacturing Equipment and Technology under Project DMETKF2013003.

\section{REFERENCES}

[1] Mulkens, J., Hinnen, P., Kubis, M., Padiy, A., and Benschop, J., "Holistic optimization architecture enabling sub-14-nm projection lithography," J. Micro/Nanolith. MEMS MOEMS 13(1), 1-10 (2014).

[2] Lam, E. Y. and Wong, A. K., "Computation lithography: virtual reality and virtual virtuality," Opt. Express 17(15), 12259-12268 (2009).

[3] Rieger, M. L., "Communication theory in optical lithography," J. Micro/Nanolith. MEMS MOEMS 11(1), 013003 (2012).

[4] Lam, E. Y. and Wong, A. K., "Nebulous hotspot and algorithm variability in computation lithography," J. Micro/Nanolith. MEMS MOEMS 9(3), 033002 (2010).

[5] Sturtevant, J., Tejnil, E., Lin, T., Schulze, S., Buck, P., Kalk, F., Nakagawa, K., Ning, G., Ackmann, P., Gans, F., et al., "Impact of 14-nm photomask uncertainties on computational lithography solutions," $J$. Micro/Nanolith. MEMS MOEMS 13(1), 011004 (2014).

[6] Tejnil, E., Hu, Y., Sahouria, E., Schulze, S., Tian, M. J., and Guo, E., "Advanced mask process modeling for 45-nm and 32-nm nodes," Proc. SPIE 6924, 69243H (2008).

[7] Jia, N., Yang, S.-H., Kim, S., and Choi, J., "Study of lens heating behavior and thick mask effects with a computational method," Proc. SPIE 9052, 905209 (2014).

[8] Rudolph, O. H., Evanschitzky, P., Erdmann, A., Bär, E., and Lorenz, J., "Rigorous electromagnetic field simulation of the impact of photomask line-edge and line-width roughness on lithographic processes," $J$. Micro/Nanolith. MEMS MOEMS 11(1), 013004 (2012).

[9] Mack, C. A., "Line-edge roughness and the impact of stochastic processes on lithography scaling for Moore's law," Proc. SPIE 9180, 91890D (2014).

[10] Mack, C., [Fundamental Principles of Optical Lithography: The Science of Microfabrication], Wiley, Chichester (2007).

[11] Pang, L., Xiao, G., Tolani, V., Hu, P., Cecil, T., Dam, T., Baik, K.-H., and Gleason, B., "Considering MEEF in inverse lithography technology (ILT) and source mask optimization (SMO)," Proc. SPIE 7122, $71221 \mathrm{~W}(2008)$.

[12] Lam, E. Y. and Wu, X., "Impact of photomask shape uncertainties on computational lithography," in [Semiconductor Technology International Conference (CSTIC)], submitted (2016). 
[13] Lv, W., Liu, S., Xia, Q., Wu, X., Shen, Y., and Lam, E. Y., "Level-set-based inverse lithography for mask synthesis using the conjugate gradient and an optimal time step," J. Vac. Sci. Technol. B 31, 041605 (2013).

[14] Chen, S., Chen, W., and Lee, S., "Level set based robust shape and topology optimization under random field uncertainties," Struct. Multidiscip. O. 41(4), 507-524 (2010).

[15] Wu, X., Liu, S., Lv, W., and Lam, E. Y., "Robust and efficient inverse mask synthesis with basis function representation," J. Opt. Soc. Am. A 31(12), B1-B9 (2014).

[16] Wu, X., Liu, S., Lv, W., and Lam, E. Y., "Sparse nonlinear inverse imaging for shot count reduction in inverse lithography," Opt. Express 23(21), 26919-26931 (2015). 